九州大学学術情報リポジトリ

Kyushu University Institutional Repository

\title{
STRATIFIED RANDOM SAMPLING ; RANK CORRELATION COEFFICIENTS, TESTS OF INDEPENDENCE AND RANDOM CONFIDENCE INTERVALS
}

Yanagawa, Takashi

Department of Mathematics, Kyushu University

https://doi.org/10.5109/13069

出版情報: 統計数理研究. 15 (3/4), pp. 21-30，1973-03. Research Association of Statistical Sciences

バージョン :

権利関係 : 


\title{
STRATIFIED RANDOM SAMPLING; RANK CORRELATION COEFFICIENTS, TESTS OF INDEPENDENCE AND CONFIDENCE INTERVALS
}

By

\author{
Takashi YanAGAWA*
}

(Received November 25, 1971)

\section{§. Introduction}

The problem of giving reasonable measures of association when a population is stratified was first investigated by Aoyama [1]. Recently Wakimoto [3] considered the problem more extensively. He gave an estimator of the correlation coefficient based on a stratified random sample and showed it to be superior to the one given by Aoyama.

The purpose of this paper is to propose new measures of association, test of independence and confidence intervals based on a stratified random sample. These measures are stratified version of Kendall and Speaman rank correlation coefficients. Throughout this paper we assume that each size of stratum is sufficiently large compared with that of sample taken from it so that the finite correction term may be neglected. In section 2 measures of association, tests of independence and confidence intervals based on a stratified random sample is given. A stratified version of Kendall rank correlation coefficient is discussed in section 2.1 and then in section 2.2 the one of Speaman type is discussed. In section 3 gains in efficiency due to stratification is demonstrated in the case of proportional allocation by comparing proposed measures with respect to Kendall and Speaman rank correlation coefficient based on a simple random sample.

\section{$\S 2$. Measures of association, tests of independence and confidence intervals}

Suppose that the population $\pi$ with two-dimensional distribution function $F(x)$ is classified into $L$ strata $\left\{\pi_{1}, \cdots, \pi_{L}\right\}$, which may be overlapping, in such a way that the two-dimensional distribution function $F_{i}(x)$ corresponds to the $i$-th stratum $\pi_{i}$ satisfies the relation

$$
F(x)=\sum_{i=1}^{L} w_{i} F_{i}(x) \quad \text { for all } x
$$

where $w_{i}$ is a known weight of $F_{i}$ such that $0 \leqq w_{i} \leqq 1, \sum_{i=1}^{L} w_{i}=1$.

* Department of Mathematics, Kyushu University, Fukuoka. 
Now suppose we have a sampling plan to take a two-dimensional random sample: $\left(X_{i 1}^{(1)}, X_{i 1}^{(2)}\right), \cdots,\left(X_{i n_{i}}^{(1)}, X_{i n_{i}}^{(2)}\right)$ from the $i$-th stratum $\pi_{i}(i=1 \sim L)$. We assume $n=\sum_{i=1}^{L} n_{i}$ the total sample size. Let $\left(X_{1}^{(1)}, X_{1}^{(2)}\right), \cdots,\left(X_{n}^{(1)}, X_{n}^{(2)}\right)$ be a two-dimensional simple random sample of size $n$ from the population $\pi$.

We need the following definitions and notations in the sequel.

$$
\begin{aligned}
& S(u)=\left\{\begin{array}{rrr}
-1 & \text { if } u<0 \\
0 & \text { if } u=0 \\
1 & \text { if } u>0
\end{array}\right. \\
& R_{\alpha} ; \text { rank of } X_{\alpha}^{(1)} \text { among } X_{1}^{(1)}, \cdots, X_{n}^{(1)}, \\
& S_{\alpha} ; \text { rank of } X_{\alpha}^{(2)} \text { among } X_{1}^{(2)}, \cdots, X_{n}^{(2)}, \\
& R_{i \alpha} ; \operatorname{rank} \text { of } X_{i \alpha}^{(1)} \text { among } X_{i 1}^{(1)}, \cdots, X_{i n_{i}}^{(1)}, \\
& S_{i \alpha} ; \operatorname{rank} \text { of } X_{i \alpha}^{(2)} \text { among } X_{i 1}^{(2)}, \cdots, X_{i n_{i}}^{(2)}, \\
& R_{i \alpha}^{i j} ; \operatorname{rank} \text { of } X_{i \alpha}^{(1)} \text { among } X_{i 1}^{(1)}, \cdots, X_{i n_{i}}^{(1)}, X_{j 1}^{(1)}, \cdots, X_{j n_{j}}^{(1)}, \\
& S_{i \alpha}^{i j} ; \operatorname{rank} \text { of } X_{i \alpha}^{(2)} \text { among } X_{i 1}^{(2)}, \cdots, X_{i n_{i}}^{(2)}, X_{j 1}^{(2)}, \cdots, X_{j n_{j}}^{(2)} .
\end{aligned}
$$

We get following equalities.

$$
\begin{aligned}
& R_{\alpha}=\frac{n+1}{2}+\frac{1}{2} \sum_{\beta=1}^{n} S\left(X_{\alpha}^{(1)}-X_{\beta}^{(1)}\right) \\
& S_{\alpha}=\frac{n+1}{2}+\frac{1}{2} \sum_{\beta=1}^{n} S\left(X_{\alpha}^{(2)}-X_{\beta}^{(2)}\right) \\
& R_{i \alpha}=\frac{n_{i}+1}{2}+-\frac{1}{2} \sum_{\beta=1}^{n_{i}} S\left(X_{i \alpha}^{(1)}-X_{i \beta}^{(1)}\right) \\
& S_{i \alpha}=\frac{n_{i}+1}{2}+\frac{1}{2} \sum_{\beta=1}^{n_{i}} S\left(X_{i \alpha}^{(2)}-X_{i \beta}^{(2)}\right) \\
& R_{i \alpha}^{i j}-R_{i \alpha}=\frac{n_{j}}{2}+\frac{1}{2} \sum_{\beta=1}^{n_{j}} S\left(X_{i \alpha}^{(1)}-X_{j \beta}^{(1)}\right) \\
& S_{i \alpha}^{i j}-S_{i \alpha}=\frac{n_{j}}{2}+\frac{1}{2} \sum_{\beta=1}^{n_{j}} S\left(X_{i \alpha}^{(2)}-X_{j \beta}^{(2)}\right) .
\end{aligned}
$$

\subsection{Kendall's type}

Kendall's rank correlation coefficient in the simple random sampling is defined: as

$$
r_{K}=\frac{1}{n(n-1)} \sum_{\alpha \neq \beta} S\left(X_{\alpha}^{(1)}-X_{\beta}^{(1)}\right) S\left(X_{\alpha}^{(2)}-X_{\beta}^{(2)}\right) .
$$

$r_{K}$ is an unbiased estimator of

$$
\tau=\int s\left(x_{1}^{(1)}-x_{2}^{(1)}\right) S\left(x_{1}^{(2)}-x_{2}^{(2)}\right) d F\left(x_{1}\right) d F\left(x_{2}\right), \quad x_{i}=\left(x_{1}^{(i)}, x_{2}^{(i)}\right) \quad i=1,2 .
$$

In the stratified case we propose following $r_{K}^{*}$ as a measure of association, which 
belongs to the class of U-statistics considered by Yanagawa, T. and Wakimoto, $\mathrm{K}$. [5].

$$
r_{K}^{*}=\sum_{i=1}^{L} \sum_{j=1}^{L} w_{i} w_{j} t_{i j}
$$

where

$$
\begin{aligned}
& t_{i i}=\frac{1}{n_{i}\left(n_{i}-1\right)} \sum_{\alpha \neq \beta}^{n_{i}} S\left(X_{i \alpha}^{(1)}-X_{i \beta}^{(1)}\right) S\left(X_{i \alpha}^{(2)}-X_{i \beta}^{(2)}\right), \\
& t_{i j}=\frac{1}{n_{i} n_{j}} \sum_{\alpha=1}^{n_{i}} \sum_{\beta=1}^{n_{j}} S\left(X_{i \alpha}^{(1)}-X_{j \beta}^{(1)}\right) S\left(X_{i \alpha}^{(2)}-X_{j \beta}^{(2)}\right) \quad(i \neq j) .
\end{aligned}
$$

Let us put

$$
\begin{aligned}
& \varphi_{K}\left(x_{1}, x_{2}\right) \equiv S\left(x_{1}^{(1)}-x_{2}^{(1)}\right) S\left(x_{1}^{(2)}-x_{2}^{(2)}\right), \\
& \varphi_{K 1}(x)=\int S\left(x^{(1)}-x_{2}^{(1)}\right) S\left(x^{(2)}-x_{2}^{(2)}\right) d F\left(x_{2}\right), \\
& x=\left(x^{(1)}, x^{(2)}\right), \quad x_{i}=\left(x_{i}^{(1)}, x_{i}^{(2)}\right) .
\end{aligned}
$$

THEOREM 1. (i) $r_{K}^{*}$ is an unbiased estimator of $\tau$ and its variance is given by

$$
\operatorname{Var}\left[r_{K}^{*}\right]=4 \sum_{i=1}^{L} \frac{w_{i}^{2}}{n_{i}} \operatorname{Var}\left[\varphi_{K 1}\left(X_{i 1}\right)\right]+2 \sum_{i=1}^{L} \frac{w_{i}^{4}}{n_{i}\left(n_{i}-1\right)} a_{i i}+2 \sum_{i \neq j}^{L} \frac{w_{i}^{2} w_{j}^{2}}{n_{i} n_{j}} a_{i j},
$$

where

$$
a_{i \jmath}=\operatorname{Var}\left[\varphi_{K}\left(X_{i 1}, X_{j 2}\right)\right]-2 \operatorname{Cov}\left[\varphi_{K}\left(X_{i 1}, X_{j 2}\right), \varphi_{K}\left(X_{i 1}, X_{j 3}\right)\right] .
$$

(ii) Let $n \rightarrow \infty$ in such a way that $n_{i} / n \rightarrow \lambda_{i}$, where $\lambda_{i}$ is a constant such that $0<\lambda_{i}<1(i=1 \sim L)$ and $\sum_{i=1}^{L} \lambda_{i}=1$. Then $\sqrt{n}\left(r_{B}^{*}-\tau\right)$ has a normal limiting distribution with mean 0 and variance

$$
\sigma_{K}^{* 2}=4 \sum_{i=1}^{L} \frac{w_{i}^{2}}{\lambda_{i}} \operatorname{Var}\left[\varphi_{K 1}\left(X_{i 1}\right)\right] .
$$

PROOF. (i) is obtained directly by simple but some lengthy computations and (ii) comes from a slight modification of the proof of Lemma 1.2 of [5], and so we shall omit the proof.

From the theorem we immediately get following corollaries.

COROLlaRY 1. (Asymptotic optimum allocation) The infimum value of $\sigma_{K}^{* 2}$ over all $\lambda_{i}$ such that $0<\lambda_{i}<1$ and $\sum_{i=1}^{L} \lambda_{i}=1$ is

$$
\sigma_{K \cdot \text { opt. }}^{* 2}=\left(\sum_{i=1}^{L} w_{i} A_{i}\right)^{2},
$$

where

$$
A_{i}^{2}=\operatorname{Var}\left[\varphi_{K 1}\left(X_{i 1}\right)\right] .
$$

Further the optimum $\lambda_{i}^{*}$ which attains the infimum value is given by

$$
\lambda_{i}^{*}=w_{i} A_{i} / \sum_{j=1}^{L} w_{j} A_{j} .
$$

COROLlaRY 2. (Asymptotic proportional allocation) When $\lambda_{i}=w_{i}$ for all $i=1 \sim L$ we get a proportional allocation and $\sigma_{K}^{* 2}$ becomes 


$$
\sigma_{K \text {.prop. }}^{* 2}=4\left\{\int \varphi_{K 1}^{2} d F-\sum_{i=1}^{L} w_{i}\left(\int \varphi_{K 1} d F_{i}\right)^{2}\right\} .
$$

Since $F$ and $F_{i}$ are assumed to be unknown and from (2.15)

$$
A_{i}^{2}=\int\left(\varphi_{K 1}-\int \varphi_{K 1} d F_{i}\right)^{2} d F_{i}
$$

then $A_{i}^{2}$ is also unknown. Thus for the problem of testing and confidence interval we need its consistent estimator, which is given by the following Lemma.

LeMma 1. Put

$$
\begin{gathered}
g\left(x_{i \alpha}\right)=\frac{w_{i}}{n_{i}-1} \sum_{\beta \neq \alpha}^{n_{j}} \varphi\left(x_{i \alpha}, x_{i \beta}\right)+\sum_{j \neq i}^{L} \frac{w_{j}}{n_{j}} \sum_{\beta=1}^{n_{j}} \varphi\left(x_{i \alpha}, x_{j \beta}\right), \\
\bar{g}_{i}=\sum_{\alpha=1}^{n_{i}} g\left(x_{i \alpha}\right) / n_{i},
\end{gathered}
$$

and

$$
S_{i}^{2}=\sum_{\alpha=1}^{n_{i}}\left[g\left(x_{i \alpha}\right)-\bar{g}_{i}\right]^{2} /\left(n_{i}-1\right),
$$

then for $A_{i}^{2}$, given in (2.15), we get

and

$$
E\left[S_{i}^{2}\right] \longrightarrow A_{i}^{2} \quad \text { as } n \longrightarrow \infty \quad(i=1 \sim L)
$$

(iii) $\quad S_{i}^{2} \longrightarrow A_{i}^{2} \quad$ in prob. as $n \longrightarrow \infty \quad(i=1 \sim L)$.

Proof. See Yanagawa, T. [4] Lemma 2.

Corollary 3. Put

$$
T_{K, n}^{*}=\sqrt{n}\left(r_{K}^{*}-\tau\right) / 2\left(\sum_{i=1}^{L} w_{i}^{2} S_{i}^{2} / \lambda_{i}\right)^{1 / 2} .
$$

then under the assumption of Theorem $1 T_{R, n}^{*}$ has a standard normal limiting distribution.

Proof. Proof is immediately obtained from Theorem 1 and Lemma 1.

From the lemma and corollaries we get following applications.

Application 1. An estimator of optimum $\lambda_{i}^{*}$ is given as $\hat{\lambda}_{i}^{*}=w_{i} S_{i} / \sum_{i=1}^{L} w_{i} S_{i}$.

Application 2. Consider the test of independence such that

$$
\begin{aligned}
H ; \quad & F\left(x^{(1)}, x^{(2)}\right)=F\left(x^{(1)}, \infty\right) \cdot F\left(\infty, x^{(2)}\right) \\
A H ; & F\left(x^{(1)}, x^{(2)}\right) \neq F\left(x^{(1)}, \infty\right) \cdot F\left(\infty, x^{(2)}\right) .
\end{aligned}
$$

Since under the hypothesis we get $\tau=0, T_{R, n}^{*}$ given by (2.21) with $\tau=0$ is proposed as a test statistic of the hypothesis based on the stratified random sample. Especially in the case of proportional allocation since we get $\tau=0$ and $\varphi_{K 1}(x)=$

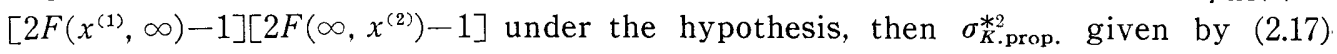
becomes

$$
\sigma_{K . \text { prop. }}^{* 2}=4\left[1 / 9-\sum_{i=1}^{L} w_{i}\left(\int \varphi_{K 1} d F_{i}\right)^{2}\right] .
$$

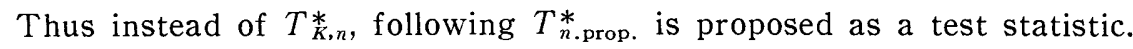

$$
T_{n . \text { prop. }}^{*}=\sqrt{n} r_{K}^{*} / 2\left[1 / 9-\sum_{i=1}^{L} w_{i} \bar{g}_{i}^{2}\right]^{1 / 2}
$$


where $\bar{g}_{i}$ is given by (2.19).

Application 3. Approximate confidence intervals of confidence coefficient $(1-\alpha)$ is given as follows.

$$
\begin{aligned}
& \sqrt{n} r_{R}^{*}-2 k(\alpha / 2)\left(\sum_{i=1}^{L} w_{i}^{2} S_{i}^{2} / \lambda_{i}\right)^{1 / 2} \leqq \tau \\
\leqq & \sqrt{n} r_{R}^{*}-2 k(\alpha / 2)\left(\sum_{i=1}^{L} w_{i}^{2} S_{i}^{2} / \lambda_{i}\right)^{1 / 2},
\end{aligned}
$$

where $k(\alpha)$ is an upper $\alpha$ percentile point of a standard normal distribution.

\subsection{Speaman's type in the case of proportional allocation}

In the simple random sampling Speaman's rank correlation coefficient is

$$
r_{S}=\frac{12}{n^{3}-n} \sum_{\alpha=1}^{n}\left(R_{\alpha}-\frac{n+1}{2}\right)\left(S_{\alpha}-\frac{n+1}{2}\right)
$$

It is an unbiased estimator of

$$
\begin{array}{r}
\rho=\frac{3}{n+1}\left[(n-2) \int S\left(x_{1}^{(1)}-x_{2}^{(1)}\right) S\left(x_{1}^{(2)}-x_{3}^{(2)}\right) \prod_{i=1}^{3} d F\left(x_{i}\right)\right. \\
\left.+\int S\left(x_{1}^{(1)}-x_{2}^{(1)}\right) S\left(x_{1}^{(2)}-x_{2}^{(2)}\right) \prod_{i=1}^{2} d F\left(x_{i}\right)\right] .
\end{array}
$$

In this section we shall give a stratified version of Speaman's rank correlation coefficient. It is quite complicated and that we shall consider it only the case of proportional allocation. Through similar discussions as previous section we can get an unbiased estimator of $\rho$ based on U-statistics. But here we shall concentrate our considerations to estimators based on ranks and that we shall propose following $r_{s}^{*}$ as a measure of association in the case of proportional allocation.

$$
r_{s}^{*}=\frac{12}{n^{2}(n+1)}-\sum_{i=1}^{L} \sum_{\alpha=1}^{n_{i}}\left[\sum_{j \neq i}^{L} R_{i \alpha}^{i j}-(L-2) R_{i \alpha}-\frac{n+1}{2}\right]\left[\sum_{K \neq i}^{L} S_{i \alpha}^{i k}-(L-2) S_{i \alpha}-\frac{n+1}{2}\right] .
$$

Put

$$
\begin{aligned}
& \varphi_{s}\left(x_{1}, x_{2}, x_{3}\right)=S\left(x_{1}^{(1)}-x_{2}^{(1)}\right) S\left(x_{1}^{(2)}-x_{3}^{(2)}\right), \\
& \phi\left(x_{1}, x_{2}, x_{3}\right)=\Sigma^{\prime} \varphi_{s}\left(x_{i_{1}}, x_{i_{2}}, x_{i_{3}}\right) / 2,
\end{aligned}
$$

where the sum $\Sigma^{\prime}$ is taken over all permutation $\left(i_{1}, i_{2}, i_{3}\right)$ of $(1,2,3)$. Then we get the following theorem.

THEOREM 2. (i) $E\left(r_{s}^{*}\right)=\rho+B /(n+1)$, where

$$
B=3 \sum_{i=1}^{L} w_{i}\left\{\int \phi\left(x_{1}, x_{2}, x_{3}\right) \prod_{j=1}^{2} d F_{i}\left(x_{j}\right) d F\left(x_{3}\right)-2 \int \phi\left(x_{1}, x_{2}, x_{3}\right) \prod_{j=1}^{3} d F\left(x_{j}\right)\right\} .
$$

(ii) Let $n \rightarrow \infty$ in such a way that $n_{i} / n \rightarrow w_{i}$ for all $i=1 \sim L$, then $\sqrt{n}\left(r_{s}^{*}-\rho\right)$ has a normal limiting distribution with mean 0 and variance

$$
\sigma_{s}^{* 2}=9 \sum_{i=1}^{L} w_{i} \int\left[\psi_{s}(x)-\rho_{i} \cdot\right]^{2} d F_{i}(x)
$$

where 


$$
\psi_{s}(x)=\int \phi\left(x, x_{2}, x_{3}\right) \prod_{i=2}^{3} d F\left(x_{i}\right)
$$

and

$$
\rho_{i}=\int \psi_{s}(x) d F_{i}(x) .
$$

Proof. As the proof of the theorem is complicated, we shall give it in the appendix.

Now let us consider the test of independence. Under the hypothesis we get $\rho=0$ and $\phi_{s}(x)=\left[2 F\left(x^{(1)}, \infty\right)-1\right]\left[2 F\left(\infty, x^{(2)}-1\right]\right.$, then it follows that $\sigma_{s}^{* 2}=9 \sigma_{K, \text { prop }}^{* 2} / 4$, where $\sigma_{R \text {.prop. }}^{* 2}$ is given by (2.17). Thus we get the following test statistic for the test of independence.

$$
T_{s, n}^{*}=\sqrt{n} r_{s}^{*} / 3\left[1 / 9-\sum_{i=1}^{L} w_{i} \bar{g}_{i}^{2}\right]^{1 / 2},
$$

where $\bar{g}_{i}$ is given by (2.19).

\section{§3. Gain in efficiency due to stratification}

In this section we shall consider the gain in efficiency due to stratification. Let $X_{\alpha}=\left(X_{\alpha}^{(1)}, X_{\alpha}^{(2)}\right), \alpha=1,2,3$ be i. i. d. random variables with distribution function. $F(x), X_{i \beta}=\left(X_{i \beta}^{(1)}, X_{i \beta}^{(2)}\right)$ be i. i. d. random variables with distribution function $F_{i}(x)$, $i=1 \sim L$, and let us put

$$
\varphi_{K}^{j}(x)=\int \varphi_{K}(x, y) d F_{j}(y), \quad j=1 \sim L .
$$

LEMMA 2.

$$
\begin{aligned}
& \operatorname{Var}\left[\varphi_{K}\left(X_{1}, X_{2}\right)\right] \geqq 2 \operatorname{Var}\left[\varphi_{K 1}\left(X_{1}\right)\right] . \\
& \sum_{i, j}^{L} w_{i} w_{j}\left\{\operatorname{Cov}\left[\varphi_{K}\left(X_{i 1}, X_{j 2}\right), \varphi_{K}\left(X_{i 1}, X_{j 3}\right)\right]-\operatorname{Var}\left[\varphi_{K 1}\left(X_{i 1}\right)\right]\right\} \\
& \quad=\sum_{i, j}^{L} w_{i} w_{j} E\left\{\left[\varphi_{K}^{j}\left(X_{i 1}\right)-E \varphi_{K}^{i}\left(X_{i 1}\right)\right]-\left[\varphi_{K 1}\left(X_{i 1}\right)-E \varphi_{K 1}\left(X_{i 1}\right)\right]\right\}^{2} .
\end{aligned}
$$

Proof. (i) See Fraser [2], p. 227, Theorem 5.2. (ii) Since

$$
\begin{aligned}
\operatorname{Cov} & {\left[\varphi_{K}\left(X_{i 1}, X_{j 2}\right), \varphi_{K}\left(X_{i 1}, X_{j 3}\right)\right]-\operatorname{Var}\left[\varphi_{K 1}\left(X_{i 1}\right)\right] } \\
& =E\left[\varphi_{K}^{j}\left(X_{i 1}\right)-E \varphi_{K}^{j}\left(X_{i 1}\right)\right]^{2}-E\left[\varphi_{K 1}\left(X_{i 1}\right)-E \varphi_{K 1}\left(X_{i 1}\right)\right]^{2},
\end{aligned}
$$

and

$$
\begin{gathered}
\sum_{i, j} w_{i} w_{j} E\left\{\varphi_{K}^{j}\left(X_{i 1}\right)-E \varphi_{K}^{j}\left(X_{i 1}\right)\right]\left[\varphi_{K 1}\left(X_{i 1}\right)-E \varphi_{K 1}\left(X_{i 1}\right)\right] \\
=\sum_{i} w_{i} E\left[\varphi_{K 1}\left(X_{i 1}\right)-E \varphi_{K 1}\left(X_{i 1}\right)\right]^{2},
\end{gathered}
$$

then we get

$$
\begin{aligned}
\sum_{i, j} w_{i} w_{j}\left\{\operatorname{Cov}\left[\varphi_{K}\left(X_{i 1}, X_{j 2}\right), \varphi_{K}\left(X_{i 1}, X_{j 3}\right)\right]-\operatorname{Var}\left[\varphi_{K 1}\left(X_{i 1}\right)\right]\right\} \\
=\sum_{i, j} w_{i} w_{j} E\left\{\left[\varphi_{K}^{j}\left(X_{i 1}\right)-E \varphi_{K}^{j}\left(X_{i 1}\right)\right]-\left[\varphi_{K 1}\left(X_{i 1}\right)-E \varphi_{K 1}\left(X_{i 1}\right)\right]\right\}^{2} .
\end{aligned}
$$

THEOREM 3. Suppose the proportional allocation. (i) Put 


$$
\begin{aligned}
P= & \sum_{i=1}^{L} w_{i}\left[E \varphi_{K 1}\left(X_{i 1}\right)-E \varphi_{K 1}\left(X_{1}\right)\right]^{2}, \\
Q= & \sum_{i, j}^{L} w_{i} w_{j}\left[E \varphi_{K}\left(X_{i 1}, X_{j 2}\right)-E \varphi_{K}\left(X_{1}, X_{2}\right)\right]^{2}-2 \sum_{i=1}^{L} w_{i}\left[E \varphi_{K 1}\left(X_{i 1}\right)-E \varphi_{K 1}\left(X_{1}\right)\right]^{2} \\
& +2 \sum_{i, j}^{L} w_{i} w_{j}\left\{\operatorname{Cov}\left[\varphi_{K}\left(X_{i 1}, X_{j 2}\right), \varphi_{K}\left(X_{i 1}, X_{j 3}\right)\right]-\operatorname{Var}\left[\varphi_{K 1}\left(X_{i 1}\right)\right]\right\},
\end{aligned}
$$

then we get

$$
\operatorname{Var}\left[r_{K}\right]-\operatorname{Var}\left[r_{K}^{*}\right]=\frac{4}{n} P+\frac{2}{n(n-1)} Q+O\left(\frac{1}{n^{3}}\right)
$$

and

$$
\frac{4}{n} P+\frac{2}{n(n-1)} Q \geqq 0 \text {. }
$$

$$
E\left[r_{s}-\rho\right]^{2}-E\left[r_{s}^{*}-\rho\right]^{2}=\frac{9}{n} \sum_{i=1}^{L} w_{i}\left[\int \phi_{s}(x) d F_{i}(x)-\rho\right]^{2}+0\left(\frac{1}{n^{2}}\right) .
$$

Proof. (i) Variance of Kendall's rank correlation coefficient $r_{K}$ is given by'

$$
\operatorname{Var}\left[r_{K}\right]=\frac{4(n-2)}{n(n-1)} \operatorname{Var}\left[\varphi_{K 1}\left(X_{1}\right)\right]+\frac{2}{n(n-1)} \operatorname{Var}\left[\varphi_{K}\left(X_{1}, X_{2}\right)\right] \text {. }
$$

Substituting (2.1), it follows

$$
\begin{aligned}
\operatorname{Var}\left[r_{K}\right]= & \frac{4}{n}\left\{\sum_{i=1}^{L} w_{i} \operatorname{Var}\left[\varphi_{K 1}\left(X_{i 1}\right)\right]+\sum_{i=1}^{L} w_{i}\left[E \varphi_{K 1}\left(X_{i 1}\right)-E \varphi_{K 1}\left(X_{1}\right)\right]^{2}\right\} \\
+ & \frac{2}{n(n-1)}\left\{\sum_{i, j}^{L} w_{i} w_{j} \operatorname{Var}\left[\varphi_{K}\left(X_{i 1}, X_{j 2}\right)\right]\right. \\
& +\sum_{i, j}^{L} w_{i} w_{j}\left[E \varphi_{K}\left(X_{i 1}, X_{j 2}\right)-E \varphi_{K}\left(X_{1}, X_{2}\right)\right]^{2} \\
& \left.-2 \sum_{i=1}^{L} w_{i}\left\{\operatorname{Var}\left[\varphi_{K 1}\left(X_{i 1}\right)\right]+\left[E \varphi_{K 1}\left(X_{i 1}\right)-E \varphi_{K 1}\left(X_{1}\right)\right]^{2}\right\}\right\} .
\end{aligned}
$$

Thus from (2.12) we get

$$
\begin{aligned}
& \operatorname{Var}\left[r_{K}\right]-\operatorname{Var}\left[r_{K}^{*}\right]=\frac{4}{n} \sum_{i=1}^{L} w_{i}\left[E \varphi_{K 1}\left(X_{i 1}\right)-E \varphi_{K 1}\left(X_{1}\right)\right]^{2} \\
& +\frac{2}{n(n-1)}\left\{\sum_{i, j}^{L} w_{i} w_{j}\left[E \varphi_{K}\left(X_{i 1}, X_{j 2}\right)-E \varphi_{K}\left(X_{1}, X_{2}\right)\right]^{2}\right. \\
& \quad-2 \sum_{i=1}^{L} w_{i}\left[E \varphi_{K 1}\left(X_{i 1}\right)-E \varphi_{K 1}\left(X_{1}\right)\right]^{2} \\
& \left.\quad+2 \sum_{i, j}^{L} w_{i} w_{j}\left(\operatorname{Cov}\left[\varphi_{K}\left(X_{i 1}, X_{j 2}\right), \varphi_{K}\left(X_{i 1}, X_{j 3}\right)\right]-\operatorname{Var}\left[\varphi_{1}\left(X_{i 1}\right)\right]\right)\right\} \\
& +\frac{2}{n^{2}}\left\{\sum_{i, j}^{L} \frac{w_{i} w_{j}}{n-1}\left(\operatorname{Var}\left[\varphi_{K}\left(X_{i 1}, X_{j 2}\right)\right]-2 \operatorname{Cov}\left[\varphi_{K}\left(X_{i 1}, X_{j 2}\right), \varphi_{K}\left(X_{i 1}, X_{j 3}\right)\right]\right)\right. \\
& \left.\quad-\sum_{i=1}^{L} \frac{w_{i}^{2}}{n_{i}-1}\left(\operatorname{Var}\left[\varphi_{K}\left(X_{i 1}, X_{j 2}\right)\right]-2 \operatorname{Cov}\left[\varphi_{K}\left(X_{i 1}, X_{j 2}\right), \varphi_{K}\left(X_{i 1}, X_{j 3}\right)\right]\right)\right\} \\
& =\frac{4}{n} P+\frac{2}{n(n-2)} Q+0\left(\frac{1}{n^{3}}\right) .
\end{aligned}
$$


From Lemma $2, \frac{4}{n} P+\frac{2}{n(n-1)} Q \geqq 0$ is clear. Thus we get (i).

(ii) From Theorem 2

$$
\begin{aligned}
E\left[r_{s}-\rho\right]^{2}-E\left[r_{s}^{*}-\rho\right]^{2} & =\operatorname{Var}\left[r_{s}\right]-\operatorname{Var}\left[r_{s}^{*}\right]+0\left(1 / n^{2}\right) \\
& =\operatorname{Var}[K]-\operatorname{Var}\left[K^{*}\right]+0\left(1 / n^{2}\right),
\end{aligned}
$$

where $K=6 \Sigma^{\prime \prime} \phi\left(X_{\alpha}, X_{\beta}, X_{\gamma}\right) / n(n-1)(n-2)$, the summation $\Sigma^{\prime \prime}$ being over all combinations $(\alpha, \beta, \gamma)$ such that $1 \leqq \alpha<\beta<\gamma \leqq n$ and $K^{*}$ is given by (2) in the appendix. Since $K$ and $K^{*}$ are U-statistics, and through some similar discussions as (i), we get from the proof of Theorem 2 that

$$
\operatorname{Var}[K]=\frac{9}{n} \operatorname{Var}\left[\phi_{s}\left(X_{1}\right)\right]+0\left(\frac{1}{n^{2}}\right)
$$

and

$$
\operatorname{Var}\left[K^{*}\right]=\frac{9}{n} \sum_{i=1}^{L} w_{i} \int\left[\psi_{s}(x)-\rho_{i} \cdot\right]^{2} d F_{i}(x)
$$

where

$$
\rho_{i}=\int \phi_{s}(x) d F_{i}(x), \quad i=1 \sim L
$$

Thus

$$
\begin{aligned}
\operatorname{Var}[K]-\operatorname{Var}\left[K^{*}\right] & =\frac{9}{n}\left\{\sum_{i=1}^{L} w_{i} \rho_{i}^{2}--\rho^{2}\right\}+0\left(\frac{1}{n^{2}}\right) \\
& =\frac{9}{n} \sum_{i=1}^{L} w_{i}\left[\int \phi_{s}(x) d F_{i}(x)-\rho\right]^{2}+0\left(\frac{1}{n^{2}}\right) .
\end{aligned}
$$

Thus we complete the proof of the theorem.

Note. From Theorem 3 we get inequalities such that $\operatorname{Var}\left(r_{k}\right) \geqq \operatorname{Var}\left(r_{k}^{*}\right)$ and $E\left[r_{s}-\rho\right]^{2} \geqq E\left[r_{s}^{*}-\rho\right]^{2}$ asymptotically in the case of proportional allocation, which show the gain in efficiency due to stratification. Unfortunately these relations do not hold even asymptotically for arbitrary allocation. For example suppose $F_{1}=\cdots=F_{L}$, which is considered as a "bad stratification", then we get the inverse inequality $\sigma_{K}^{2} \leqq \sigma_{K, \text { prop., }}^{* 2}$ where equality holds only when $\lambda_{i}=w_{i}$ for all $i=1 \sim L$.

\section{References}

1 Aoyama, H. (1954): A study of the stratified random sampling, Ann. Inst. Stat. Math., $6,1-36$.

[2] Fraser, D. A.S. (1957): Nonparpmetric Methods in Statistic. John Wiley \& Sons, Inc.

[3] WaKimoto, K. (1971): Stratified random samaling (III); Estimation of the correlation coefficient. Ann. Inst. Stat. Math., 23, pp. 339-353.

[4] YANAGAwA, T. (1972): Stratified random sampling; Testing hypotheses based on $U$. statistics. Memoirs of the Faculty of Science, Kyushu University. Series A, Vol. 26, No. 2, pp. 361-379.

$5]$ YanAgAwA, T. and WAkimoto, K. (1972): Estimation of some functional of population distribution based on a stratified random sampling. Ann. Inst. Stat. Math., Vol. 24, No. 1, pp. 137-151. 
Appendix. Proof of Theorem 2. (i) Put

$$
\rho_{1}=3 \int \varphi\left(x_{1}, x_{2}, x_{3}\right) \prod_{i=1}^{3} d F\left(x_{i}\right),
$$

then $\rho$ can be written as follows

$$
\rho=\left[(n-2) \rho_{1}+3 \tau\right] /(n+1),
$$

where $\tau$ is given in section 2.1. From Yanagawa, T. and Wakimoto, K. [5] U. M. V. unbiased estimator of $\rho_{1}$ based on stratified random sample is given as following $K^{*}$.

$$
K^{*}=A_{1}+A_{2}+A_{3},
$$

where

$$
\begin{aligned}
& A_{1}=6 \sum_{i=1}^{L} w_{i}^{3} \sum_{\alpha<\beta<\gamma}^{n_{i}} \phi\left(x_{i \alpha}, x_{i \beta}, x_{i \gamma}\right) / n_{i}\left(n_{i}-1\right)\left(n_{i}-2\right), \\
& A_{2}=6 \sum_{i=1}^{L} w_{i}^{2} w_{j} \sum_{\alpha<\beta}^{n_{i}} \sum_{\gamma=1}^{n_{j}} \phi\left(x_{i \alpha}, x_{i \hat{\gamma}}, x_{j \gamma}\right) / n_{i}\left(n_{i}-1\right) n_{j}
\end{aligned}
$$

and

$$
A_{3}=\sum_{\substack{i \neq j \neq k \\ i \neq k}}^{L} w_{i} w_{j} w_{k} \sum_{\alpha=1}^{n_{i}} \sum_{\beta=1}^{n_{j}} \sum_{\gamma=1}^{n_{k}} \phi\left(x_{i \alpha}, x_{j \beta}, x_{k i}\right) .
$$

By (2.25), (2.26), (2.2), (2.3), (2.4), (2.5), (2.6) and (2.7) and using the relation $n_{i}=w_{i} n$ for all $i=1 \sim L$ (proportional allication), we get

$$
\begin{aligned}
(n-2) A_{1}= & 3 \sum_{i=1}^{L} \frac{w_{i}^{2}}{n_{i}\left(n_{i}-1\right)} \sum_{\substack{\alpha \neq \hat{\beta} \neq \gamma \\
\alpha \neq \beta}}^{n_{i}} \varphi\left(x_{i \alpha}, x_{i \beta}, x_{i r}\right)+A_{11}, \\
(n-2) A_{2}= & 3 \sum_{i \neq j}^{L} w_{i} w_{j} \sum_{\alpha=1}^{n_{j}} \sum_{\beta=j}^{n_{j}} \varphi\left(x_{i \alpha}, x_{j \beta}, x_{j r}\right) / n_{i} n_{j} \\
& +3 \sum_{i \neq j}^{L} w_{i} w_{j} \sum_{\alpha=1}^{n_{j}}\left\{\left[2 R_{i \alpha}-\left(n_{i}+1\right)\right]\left[2\left(S_{i \alpha}^{i j}-S_{i \alpha}\right)-n_{j}\right]\right. \\
& \left.+\left[2\left(R_{i \alpha}^{i j}-R_{i \alpha}\right)-n_{j}\right]\left[2 S_{i \alpha}-\left(n_{i}+1\right)\right]\right\} / n_{i} n_{j}+A_{21} .
\end{aligned}
$$

and

$$
(n-2) A_{3}=3 \sum_{\substack{i \neq j \neq k \\ i \neq k}}^{L} \frac{w_{i} w_{j}}{n_{i} n_{j}} \sum_{\alpha=1}^{n_{i}}\left[2\left(R_{i \alpha}^{i j}-R_{i \alpha}\right)-n_{j}\right]\left[2\left(S_{i \alpha}^{i j}-S_{i \alpha}\right)-n_{k}\right]-A_{31},
$$

where

and

$$
\begin{aligned}
A_{11}= & 6 \sum_{i=1}^{L} w_{i}^{2}\left(1-w_{i}\right) \sum_{\substack{\alpha \neq \beta \neq \gamma \\
\alpha \neq \gamma}}^{n_{i}} \varphi\left(x_{i \alpha}, x_{i \beta}, x_{i \gamma}\right) / n_{i}\left(n_{i}-1\right)\left(n_{i}-2\right), \\
A_{21}= & 3 \sum_{i \neq j}^{L} w_{i} w_{j}\left(1-2 w_{i}\right) \sum_{\gamma=1}^{n_{j}} \sum_{\alpha \neq \beta}^{n_{i}}\left[\varphi\left(x_{i \alpha}, x_{i \beta}, x_{i \gamma}\right)\right. \\
& \left.+\varphi\left(x_{i \beta}, x_{j \gamma}, x_{i \alpha}\right)+\varphi\left(x_{j i}, x_{i \alpha}, x_{i \beta}\right)\right] / n_{i}\left(n_{i}-1\right) n_{j}
\end{aligned}
$$

$$
A_{31}=6 \sum_{\substack{i \neq j \neq k \\ j \neq k}}^{L} w_{i} w_{j} w_{k} \sum_{\alpha=1}^{n_{i}} \sum_{\beta=1}^{n_{j}} \sum_{\gamma=1}^{n_{k}} \varphi\left(x_{i \alpha}, x_{j \beta}, x_{k i}\right) / n_{i} n_{j} n_{k} .
$$

Note that $\left[(n-2) K^{*}+3 r_{k}^{*}\right] /(n+1)$ is an unbiased estimator of $\rho$. 
Now

where

$$
\begin{aligned}
(n-2) K^{*}+3 r_{k}^{*}= & 3 \sum_{i=1}^{L} \frac{w_{i}^{2}}{n_{i}\left(n_{i}-1\right)} \sum_{\alpha=1}^{n_{i}}\left[2 R_{i \alpha}-\left(n_{i}+1\right)\right]\left[2 S_{i \alpha}-n_{i}\left(n_{i}+1\right)\right] \\
& +3 \sum_{i \neq j}^{L} w_{i} w_{j} \sum_{\alpha=1}^{n_{i}}\left[2\left(R_{i \alpha}^{i j}-R_{i \alpha}\right)-n_{j}\right]\left[2\left(S_{i \alpha}^{i j}-S_{i \alpha}\right)-n_{j}\right] / n_{i} n_{j} \\
& +3 \sum_{i \neq j}^{L} w_{i} w_{j} \sum_{\alpha=1}^{n_{j}}\left\{\left[2 R_{i \alpha}-\left(n_{i}+1\right)\right]\left[2\left(S_{i \alpha}^{i j}-S_{i \alpha}\right)-n_{j}\right]\right. \\
& \left.+\left[2\left(R_{i \alpha}^{i j}-R_{i \alpha}\right)-n_{j}\right]\left[2 S_{i \alpha}-\left(n_{i}+1\right)\right]\right\} / n_{i} n_{j} \\
& +\sum_{i \neq j \neq k}^{L} w_{i} w_{j} \sum_{\alpha=1}^{n_{i}}\left[2\left(R_{i \alpha}^{i j}-R_{i \alpha}\right)-n_{j}\right]\left[2\left(S_{i \alpha}^{i k}-S_{i \alpha}\right)-n_{k}\right] / n_{i} n_{j} \\
& +A_{11}+A_{21}-A_{31} \\
= & \frac{12}{n^{2}} \sum_{i=1}^{L} \sum_{\alpha=1}^{n_{i}}\left\{\sum_{j \neq i}^{L} R_{i \alpha}^{i j}-(L-2) R_{i \alpha}-\frac{n+1}{2}\right\}\left\{\sum_{K \neq i}^{L} S_{i \alpha}^{i k}-(L-2) S_{i \alpha}-\frac{n+1}{2}\right\} \\
& +A_{11}+A_{21}-A_{31}+A_{41},
\end{aligned}
$$

$$
A_{41}=3 \sum_{i=1}^{L} w_{i}^{2} \sum_{\alpha=1}^{n_{i}}\left[2 R_{i \alpha}-\left(n_{i}+1\right)\right]\left[2 S_{i \alpha}-\left(n_{i}+1\right)\right] / n_{i}^{2}\left(n_{i}-1\right) .
$$

Thus we finally get the equation

$$
r_{s}^{*}=\left[(n-2) K^{*}+3 r_{K}^{*}-A_{11}-A_{21}+A_{31}-A_{41}\right] /(n+1)
$$

where $r_{s}^{*}$ is given by (2.24).

Now

$$
E\left(r_{s}^{*}\right)=\rho-E\left[A_{11}+A_{21}-A_{31}+A_{41}\right] /(n+1),
$$

and we get easily the relation $E\left[A_{11}+A_{21}-A_{31}+A_{41}\right]=B$, where $B$ is given in Theorem 2. Thus the proof of (i) is completed. (ii) Since it is easily shown that

$$
A_{11}+A_{21}-A_{31}+A_{41} \longrightarrow 0 \text { in prob. as } n \longrightarrow \infty
$$

and from Theorem $1, \sqrt{n}\left(K^{*}-\rho_{1}\right)$ has a normal limiting distribution, then from (2) the asymptotic distribution of $\sqrt{n}\left(r_{s}^{*}-\rho\right)$ is equivalent to that of $\sqrt{n}\left(K^{*}-\rho_{1}\right)$. But it is known from Yanagawa, T. and Wakimoto, K. [5] $\sqrt{n}\left(K^{*}-\rho_{1}\right)$ has, as $n \rightarrow \infty$, a normal limiting distribution with mean 0 and variance $\sigma_{s}^{* 2}$ given by (2.27). Thus we complete the proof of the theorem. 